\title{
New Neutron Sources To Be Studied
}

The final report of a panel on neutron beam sources crucial for probing condensed matter recommends urgent action to maintain Europe's leading rôle. The Study Panel, with representatives from 9 European Community countries and the Commission of the EC, was chaired by Professor J.E. Enderby of Bristol University, UK. He is a former Co-Director of the Institut LaueLangevin (ILL) in Grenoble, France, where Europe's most important reactor source for neutron scattering studies is operated by Britain, France and Germany with Spain, Switzerland and Austria as partners.

The Panel's report issued last December to the Advisory Committee of the Large Installations Programme of the CEC's Directorate for Science and R. and D. argued that the inbalance between supply and demand calls for short-term measures to efficiently exploit existing sources. Included were increased "networking" between national facilities to promote human mobility, and a continuation of the Programme that supports access to major facilities to make use of spare experimental capacity. Investments in the short term were suggested for five European sources and shortages in the long-term should start to be tackled at once, presumably because a replacement to the ILL reactor that is needed around 2010 would take about 15 years to design, build and commission.

\section{Steady-State Reactor Sources}

Structure studies using neutron scattering were initially carried out with beams from reactors designed for materials' testing. The first dedicated reactor sources came on-stream in the late 1960's and several other reactors were modified for scattering work. The $58 \mathrm{MW}$ ILL source built in 1971, and which is seeking approval for a "third breath" (troisième souffle), has a thermal neutron flux of $1.5 \times 10^{15}$ neutrons $\mathrm{cm}^{-2} \mathrm{~s}^{-1}$ with one hot ( $200 \mathrm{mV}$ energy) source, two cold ( $5 \mathrm{mV}$ energy) sources and 30 on-line instruments. Europe's newest reactor source is the modern LLB facility at CEN Saclay, France. Built in 1980, it has $25 \%$ and $20 \%$ of the ILL's power and flux, respectively, but nearly as many instruments (21). Europe also possesses 10 other smaller reactor sources with an average age of about 30 years.

With both France and the UK reasonably with provided for (the latter has ISIS, another world-class source - see below), the German community has always been concerned about its resources. The former west German capacity (equivalent to about $20 \%$ that of the ILL) will be doubled once the upgraded Hahn Meitner Institute's (HMI) reactor comes on stream (receipt of an operating permit that is due shortly was held up for many months by the former Berlin Senate). The overall situation was not improved significantly by German unification owing to the former east Germany's most important facility at Rossendorf being poorly equipped, and suffering from low safety standards and a disconcertingly large stockpile of spent fuel rods.

As it is generally more efficient to up grade ancillary equipment (monochromators, polarizers, etc.) than to simply increase beam flux, the Panel emphasized the importance of preserving medium-flux "local" facilities to allow equipment improvements for use in experiments where the highest fluxes are not required. This strategy also avoids "political problems" associated with plans to upgrade reactors (as experienced by the HMI). The Panel acknowledged, however, that environmental issues must not be ignored and it recommended the coordination of activities relevant to the whole fuel cycle, in view of restrictions in the USA on the reprocessing of spent fuel.

Nevertheless, technical obsolescence means that it is not always worthwhile to exploit modular construction features to extend the lifetimes of older reactors. Increases in the demand for beam time and the diminishing pool of expertise accompanying a contracting reactor base can best be met by consolidating resources in centralized facilities with a back-up network of "home-based" units. The Panel therefore recommended a detailed study of stateof-the art hot and cold neutron sources based on the best available reactor technology with the aim to achieve ten times the ILL flux.

\section{Pulsed Spallation Sources}

The Panel also considered pulsed sources based upon the spallation of heavy nuclei by 500 to $1500 \mathrm{MeV}$ protons. Pioneered in the USA in 1981 by Argonne Laboratory's IPNS, Europe posesses the world's best facility at the Rutherford Appleton Laboratory in the UK where ISIS recently attained a neutron flux equivalent to an average current of protons close to $100 \mu \mathrm{A}$ after five years of operation (IPNS was upgraded to an equivalent of $35 \mu \mathrm{A}$ in 1988 and a proposed Japanese $200 \mu \mathrm{A}$ source much like ISIS has not been funded).

Roughly two-thirds of the ILL and ISIS capabilities overlap, with the ILL specializing in high resolution, cold neutron work (as epitomized in the neutron spin echo technique). ISIS on the other hand produces hot beams of "epithermal" neutrons characteristic of spallation sources with energies of up to a few eV. Recent work with these beams has yielded interesting results for the dynamics of magnetic materials.

The Study Panel recommended that a new spallation facility should also be examined using the experience gained at ISIS, and at KFA Jülich during its experimentation and design work for the proposed SNQ source that lasted several years and cost DM $120 \mathrm{M}$ (the project with a goal of 5000 $\mu \mathrm{A}$ was terminated in $1984 / 5$ by the German government). There is a precedent for a joint initiative as the Panel's report refers to the International ISIS Project that included a meeting in Rapello, Italy in $\mathbf{1 9 8 6}$ at which Jülich workers participated. One should note that ISIS's efforts to attract international support has been very successful in other respects since $21 \%$ of its operating budget now comes from outside the UK. More is needed because, as the report points out in illustrating the underutilization of national facilities, operating time at ISIS could doubled without capital expenditure.

ISIS argues that the next generation of spallation sources will be based on a proton linac of the type developed in the USA for the Star Wars Initiative. The linac would give more intense pulses which can be in a sense averaged in an intensity modulated source to produce fluxes equivalent to proton currents approaching maybe fifty times today's level (i.e. $5000 \mu \mathrm{A}$ ). The characteristics of these novel modulated beams would approach those for reactors so one would enter a new operating regime in being able to use the classical triple-axis spectrometers, presently reserved for steady state reactor sources, as well as the usual timeof-flight techniques.

Generally speaking, spallation may be the better choice as reactors are approaching their limits. While the technical arguments are fairly involved, first estimates based on energy deposition and beam geometries suggest that the limit of the useful effective flux for a spallation source is 100 times that of a reactor. Thus, the SNQ aimed for ten times the ILL capacity while the Advanced Neutron (reactor) Source (ANS) proposed for Oak Ridge originally aimed for only five times, a factor that has since been reduced by about one-half.

\section{Next Steps}

The Panel recommended the formation of a small steering committee to coordinate planning for a new major facility (or facilities). Professor F. Mezei of the HMI and a leading exponent of spin echo techniques believes that such a facility would cost no more than the previous generation's $(500$. $1000 \mathrm{MECU})$. It could be financed on the HERA model where a major national (German) accelerator facility has multinational contributions.

With working panels focussing on the reactor and spallation options and involving experts from national laboratories, the steering committee could offer final recommendations as to possible technical solutions to the requirements. A feasibility study including preliminary site selection followed by an engineering study would follow.

The expected demands from users will be critical in the final analysis. The Panel noted considerable regional variations with, for example, the UK having a well-established community of 600 scientists and Spain some 70. However, the latter anticipates a $10 \%$ increase each year, stemming partly from its membership of ILL, whereas the start-up of ISIS saw its number of users, including workers from other countries, increase by rather less ( $5 \%$ each year).

The Panel is nonetheless convinced there will be a continuing and important growth in demand deriving from the unique features that make neutron beams so valuable. 